\title{
Infusión intravenosa de ketamina a bajas dosis durante cirugía de artrodesis de columna. Una estrategia en el manejo del dolor agudo posoperatorio
}

\author{
Intravenous ketamine infusión at low doses during spinal \\ arthodesis surgery. A strategy in acute postoperative pain \\ management
}

Jorman H. Tejada1,3, Jesús H. Tovar²,3, Mario G. Orozco ${ }^{4}$, Valentina Gutiérrez ${ }^{5}$, Miguel A. Pinzón ${ }^{6}$

\begin{abstract}
Introduction: Acute postoperative pain is a complex problem given the pathophysiological characteristics, increasing health costs and complications and hindering recovery[1],[2]. Spinal arthrodesis is one of the most painful surgical procedures, presenting intense and disabling pain[3],[4]. Multimodal analgesia has been the tool with the best results, based on opioids; however, the combined use of drugs and dependence on opiates are important consequences. Therefore, the analgesic behavior during the use of subanesthetic doses of ketamine is described in the patients undergoing this procedure. Methodology: A prospective case series study was conducted from JanuaryDecember 2019, with patients undergoing spinal arthrodesis who met the inclusion criteria, at the Hernando Moncaleano Perdomo University Hospital, Neiva. A univariate statistical analysis of all the variables is performed, with a joint interpretation of the results. Results: A total of 17 patients underwent surgery,
\end{abstract}

\section{Key words:}

Acute pain,

ketamine,

spinal surgery,

postoperative

MD. Neuroanestesiólogo. Coordinador Servicio de Anestesiología Hospital Universitario Hernando Moncaleano Perdomo. Neiva, Colombia.

MD. Anestesiólogo. Hospital Universitario Hernando Moncaleano Perdomo. Neiva, Colombia.

Docente Asistente Postgrado de Anestesiología y Reanimación, Universidad Surcolombiana. Neiva, Colombia.

MD. Anestesiólogo. Hospital Departamental San Antonio de Pitalito, Colombia.

Médico. Unidad de Educación Médica Hospital Universitario Hernando Moncaleano Perdomo. Neiva, Colombia.

MD. Anestesiología y Reanimación. MDc Epidemiología clínica (PEDip).

Fecha de recepción: 16 de agosto de 2020

Fecha de aceptación: 25 de noviembre de 2020

ORCID

0000-0001-9271-5262

Fuente de financiamiento: Se declara que no existe patrocinio de ninguna entidad.

Conflicto de intereses: Los autores declaran no tener ningún conflicto de interés en la investigación. 
one of whom was excluded due to complications related to the surgical material. $88 \%$ of the patients showed evaluations of mild or absent pain in at least 4 times, with a number of morphine rescues in 24 hours of 1-2 per patient and patient ambulation in $90 \%$ on the first day. Conclusions. Postoperative spinal arthrodesis patients receiving intravenous ketamine infusion-based analgesia at subanesthetic doses showed mild or absent pain scores at almost all times.

\section{RESUMEN}

Introducción: El dolor agudo postoperatorio es un problema complejo dada las características fisiopatológicas, aumentando los costos en salud y las complicaciones y dificultando la recuperación[1],[2]. La artrodesis de columna, es uno de los procedimientos quirúrgicos más dolorosos, presentando un dolor intenso e incapacitante[3],[4]. La analgesia multimodal ha sido la herramienta con mejores resultados, tomando como base los opioides; sin embargo, el uso combinado de fármacos y la dependencia a opiáceos son consecuencias importantes. Por lo anterior, se describe el comportamiento analgésico durante el uso de dosis subanestésicas de ketamina en los pacientes llevados a dicho procedimiento. Metodología: Se realiza un estudio tipo serie de casos, prospectivo de enero-diciembre de 2019, con los pacientes llevados a artrodesis de columna que cumplieron con los criterios de inclusión en el Hospital Universitario Hernando Moncaleano Perdomo, Neiva. Se realiza un análisis estadístico univariado de la totalidad de las variables, con una interpretación conjunta de los resultados. Resultados: Se intervinieron un total de 17 pacientes, uno de los cuales fue excluido por complicaciones relacionadas con el material quirúrgico. El $88 \%$ de los pacientes mostraron valoraciones de dolor leve o ausente en al menos 4 tiempos, con número de rescates de morfina en $24 \mathrm{~h}$ de 1-2 por paciente y deambulación de los pacientes en el $90 \%$ en el primer día. Conclusiones: Los pacientes posoperatorios de artrodesis de columna que recibieron analgesia basada en infusión endovenosa de ketamina a dosis subanestésicas mostraron valoraciones de dolor leve o ausente, en casi todos los tiempos.

\author{
Palabras clave: \\ Dolor agudo, \\ ketamina, \\ cirugía de columna, \\ posoperatorio
}

\author{
Lista de abreviaturas \\ - Posoperatorio (POP) \\ - Unidad de cuidados posanestésicos (UCPA) \\ - Unidad de cuidados intensivos (UCI) \\ - Escala visual análoga (EVA) \\ - Rango intercuartílico (RIC)
}

\section{Introducción}

a artrodesis de columna es un procedimiento indicado en casos de inestabilidad de la columna secundaria a diversos procesos y tiene como principal complicación la presentación de dolor agudo posoperatorio (POP) intenso o incapacitante, siendo uno de los procedimientos quirúrgicos más dolorosos que tiende a la cronificación como agravante[3],[4].
Así, el dolor agudo POP en este procedimiento se refiere como un problema de manejo complejo por sus características fisiopatológicas y que pese al impulso de nuevas estrategias terapéuticas, continúa presente desfavoreciendo la recuperación y aumentando las complicaciones[1]. El uso de ketamina como parte de la estrategia analgésica no opioide, ha demostrado evidencia favorable en la literatura[5] disminuyendo el consumo de opioides en un total acumulado especialmente en cirugías consideradas altamente dolorosas, a dosis subanestésicas, bien sea en infusión o bolo, intra y posoperatorio inmediato hasta 48 h[10]-[16], además con mejoría significativa de las náuseas y el vómito posoperatorio[11],[16]; todo ello debido no sólo a su interacción con el receptor NMDA central y medular (este último implicado en la plasticidad neuronal), sino con sistemas endógenos relacionados con 
placer (vía serotoninérgica, dopamina y receptores opioides)[5],[10]-[12],[16]-[18], mostrando ser eficaz y seguro. Estas razones han llevado a su implementación como coadyuvante analgésico en nuestros pacientes; sin embargo, se desconoce un margen descriptivo de uso y la presentación de efectos adversos con uso de dosis subanestésicas durante el intraoperatorio y posoperatorio inmediato de pacientes sometidos a artrodesis de columna; tanto en Unidad de Recuperación Postoperatoria y Hospitalización de un Hospital del sur de Colombia.

\section{Metodología}

Después de una revisión institucional y aprobación del Comité de Bioética, se realiza un estudio de serie de casos de corte prospectivo, en el Hospital Hernando Moncaleano Perdomo, en el período de enero a diciembre de 2019. Tomando una muestra a conveniencia de todos los pacientes mayores de 18 años llevados a cirugía de artrodesis de columna y que se administrara infusión intra y posoperatoria de ketamina como estrategia analgésica no opioide; excluyendo, los pacientes con contraindicaciones para el uso de ketamina, antecedente de alergia y/o intolerancia al fármaco, pacientes con limitación cognitiva o que salieran bajo ventilación mecánica, y/o que tuvieran datos incompletos de la historia clínica.

Para la recolección de los datos se diseñó una herramienta que incluía variables sociodemográficas, variables prequirúrgicas (ASA, diagnóstico, comorbilidades, tiempo de diagnóstico, presencia de dolor crónico, procedimientos previos), variables intraoperatorias (tiempo quirúrgico, vía de artrodesis y coadyuvantes analgésicos intraoperatorios) y variables posquirúrgicas (intensidad del dolor, presencia de efectos adversos, requerimiento de rescates de opioides, satisfacción del paciente). Se define como control adecuado del dolor puntuaciones menores o iguales a 4, en escala numérica del dolor o dolor leve.

Se obtuvo consentimiento informado de los pacientes participantes.

Se estableció una inducción anestésica convencional con lidocaína $2 \%$ sin epinefrina $(1 \mathrm{mg} / \mathrm{kg}$ ), propofol (1 $\mathrm{mg} / \mathrm{kg})$, rocuronio $(0,6 \mathrm{mg} / \mathrm{kg})$, infusión continua de remifentanilo $(0,2 \mathrm{mg} / \mathrm{kg} / \mathrm{min})$; mantenimiento anestésico balanceado con infusión de opioide y sevorane (manteniendo una concentración alveolar mínima o CAM 0,5). Estrategia analgésica con infusión de ketamina por bomba volumétrica, por un acceso venoso exclusivo en tres tiempos: bolo inicial durante el lavado quirúrgico a dosis de 0,3 mg/ $\mathrm{kg}$; posteriormente se inicia infusión continua a dosis de $0,15 \mathrm{mg} / \mathrm{kg} / \mathrm{h}$ durante el tiempo quirúrgico $\mathrm{y}$, finalmente, se ajusta la dosis a $0,12 \mathrm{mg} / \mathrm{kg} / \mathrm{h}$ que se mantiene durante $24 \mathrm{~h}$ del POP contadas a partir del paso del último punto de sutura a piel. Como coadyuvantes analgésicos intraoperatorios: Diclofenaco 1 $\mathrm{mg} / \mathrm{kg}$ máximo $75 \mathrm{mg}$ endovenoso (EV) o dipirona $1 \mathrm{~g}$ EV, morfina $0,1 \mathrm{mg} / \mathrm{kg}$ (administrada al final de la instrumentación de columna), profilaxis antiemética con dexametasona. Se formuló 2 mg morfina EV, para administrar sólo como estrategia de rescate en caso de dolor severo o evaluación de la intensidad del dolor mayor a 5 en escala numérica (para lo cual se realizó educación del personal de enfermería y medicina general en tal caso). Los datos fueron recogidos por un investigador designado y entrenado para tal fin.

La evaluación de la intensidad del dolor se realizó en 5 tiempos (contados a partir de la llegada del paciente a la unidad de cuidados postanestésicos (UCPA) o unidad de cuidados intensivos (UCI): en hora cero, pasada la primera hora, a las 4, 12 y 24 h, en este último tiempo se indagaba por el número de rescates de morfina, la presentación de efectos adversos y la satisfacción subjetiva del paciente con el manejo analgésico.

Se llevó a cabo el análisis univariado de la totalidad de las variables, estableciendo la frecuencia de ocurrencia de los eventos y la magnitud de los mismos a través de la distribución de frecuencias absolutas y relativas para las variables cualitativas. Se calcularon también las medidas de tendencia central, dispersión y posición de las variables cuantitativas: medianas y rango intercuartílico, para el caso de una distribución no normal de las variables, la cual se evaluó por el test de Shapiro Wilks. Una interpretación conjunta de los resultados antes descritos consolidó el análisis descriptivo.

\section{Resultados}

Durante los meses del estudio se intervinieron un total de 17 pacientes, a todos los cuales se les administró la estrategia endovenosa con ketamina. Uno de estos pacientes fue excluido debido a una complicación quirúrgica secundaria a la estimulación constante de una raíz espinal por el material quirúrgico, lo que generó dolor de difícil control y necesidad de reintervención. Obteniendo así una muestra total de 16 pacientes.

La edad media de los pacientes fue de 60 años, la mayoría de género masculino (62\%), con índice de masa corporal medio de $29 \mathrm{~kg} / \mathrm{m}^{2}$. Más de la mitad de 
los pacientes presentaban algún grado de sobrepeso y al menos una cuarta parte se encuentra en obesidad (Tabla 1).

Se encontró como diagnósticos más frecuentes las fracturas vertebrales (39\%), la compresión medu$\operatorname{lar}(19 \%)$ y las hernias discales (12\%), con tiempo de diagnósticos establecido inferior a 2 años en la cuarta parte de los sujetos. El $69 \%$ de los pacientes tenían añadida algún tipo de comorbilidad, siendo la hipertensión arterial y la diabetes las más frecuentes. El $75 \%$ de los pacientes presentaban dolor de características neuropáticas prequirúrgico y tan sólo el 31\% manifestaba un estado modulación del mismo. Sólo 3 pacientes refirieron haber tenido un manejo mínimamente invasivo del mismo (Tabla 2).

En cuanto a las variables intraoperatorias, la columna lumbosacro fue la más comúnmente intervenida (8/16), con vía de abordaje posterior en 75\%. La mitad de los pacientes fueron de clasificación ASA 3. Solo un paciente requirió transfusión de hemocomponentes y menos de la mitad requirió ácido tranexámico.

Con respecto al POP, menos de la mitad de los sujetos requirieron manejo en la $\mathrm{UCl}$. Y en cuanto a las frecuencias del manejo con adyuvantes: a 15 se les administró acetaminofén y 14 recibieron un antiinflamatorio no esteroideo y/o dipirona, sólo uno recibió corticoides, y a ningún caso se indicó neuromoduladores. La mitad de los pacientes hizo uso de rescates de opioide con un tiempo promedio de 85 min POP para la primera dosis, con un número de rescates promedio de 1 a 2 durante las $24 \mathrm{~h}$ (Figura 1).

En lo correspondiente con desenlaces generales menos de la quinta parte de los pacientes presentaron náuseas y estreñimiento, 2 pacientes presentaron un episodio emético y tan sólo 1 paciente tuvo alucinaciones (Tabla 3).

Llegando a las variables desenlace específico en lo relacionado con el control del dolor, se aprecia en términos generales un óptimo control, con valoraciones de dolor leve o ausente en el $88 \%$ en al menos
4 tiempos; la mediana de la calificación en la EVA del dolor fue de 0 , máximo de 1 en las primeras $24 \mathrm{~h}$ con un rango intercuartílico que oscila de 0 a 1 y 0 a 2; es decir, que las tres cuartas partes de la muestra presentaron calificaciones iguales o menores a 2 en todos los momentos de la evaluación. En lo referente a la satisfacción con la analgesia, se lograron puntajes óptimos con una mediana de 10 puntos con un RIC de 8,5 a 10; es decir, que al menos tres cuartas partes de los individuos lo calificaron por encima de 8/10 puntos. Finalmente, en cuanto al desenlace funcional, se apreció que casi el $90 \%$ de los pacientes lograron movilización y deambulación en el primer día POP y al segundo día ya lo hacían la totalidad de los pacientes (Tabla 4).

\section{Discusión}

Con los años múltiples definiciones de dolor han ido más allá de la percepción física, abarcando además aspectos psíquicos y sus implicaciones sobre las actividades diarias y ello a su vez, sobre el desempeño económico y la calidad de vida en la población activa[5], principalmente en aquellos con dolores complejos como el lumbar, en quienes al menos un tercio de los pacientes presentan limitaciones en sus actividades[6].

En el ambiente perioperatorio, la búsqueda de estrategias de modulación del dolor se ha convertido en un eje fundamental de la función del anestesiólogo, más aún cuando la aparición de dolor temprano genera con frecuencia persistencia del mismo entre otras consecuencias nefastas[6],[7]. En Colombia, un estudio multicéntrico publicado en 2016 que evaluó la efectividad del control del dolor POP en las UCPA en 8 clínicas del país, demostró que el 59,1\% no presentaba porcentajes aceptables de dolor controlado a las $4 \mathrm{~h}$, y molestias como náuseas eran más frecuentes en estos pacientes[8].

En una revisión sistemática sobre el uso de keta-

\begin{tabular}{lcc}
\multicolumn{3}{c}{ Tabla 1. Análisis descriptivo variables sociodemográficas } \\
\hline Variable & $\mathbf{n} / \mathbf{m}$ & \%/RIC \\
Edad (años) & 60 & $42-64$ \\
Género masculino & 10 & $62-50$ \\
Peso (kg) & 71 & $64-81$ \\
Talla (cm) & 164 & $157-167$ \\
IMC (kg/m²) & 29 & $27-30$ \\
\hline
\end{tabular}

Variables cualitativas: n, frecuencia absoluta; \%, frecuencia relativa. Variables cuantitativas: m, mediana; RIC, rango intercuartílico. 


\begin{tabular}{|c|c|c|c|}
\hline \multirow{25}{*}{ Diagnóstico } & Variable & $\mathrm{n} / \mathrm{m}$ & $\% /$ RIC \\
\hline & Fractura & 6 & 37,50 \\
\hline & Compresión medular & 3 & 18,75 \\
\hline & Hernia discal & 2 & 12,50 \\
\hline & Listesis & 2 & 12,50 \\
\hline & Canal lumbar estrecho & 1 & 6,25 \\
\hline & Escoliosis & 1 & 6,25 \\
\hline & Trauma raquimedular & 1 & 6,25 \\
\hline & Tiempo diagnóstico (años) & 0,9 & $0,4-2$ \\
\hline & Comorbilidades & 11 & 68,75 \\
\hline & Diabetes mellitus & 5 & 31,25 \\
\hline & Hipertensión arterial & 8 & 50,00 \\
\hline & Enfermedad renal crónica & 2 & 12,50 \\
\hline & Patología psiquiátrica & 3 & 18,75 \\
\hline & Otras & 4 & 25,00 \\
\hline & Dolor neuropático & 12 & 75,00 \\
\hline & Dolor controlado & 5 & 31,25 \\
\hline & Intervención invasiva previa & 0 & 0,00 \\
\hline & Intervención mínimamente invasiva previa & 3 & 18,75 \\
\hline & PreQx AINEs & 8 & 50,00 \\
\hline & PreQx opioides & 9 & 56,25 \\
\hline & PreQx neuromoduladores & 3 & 18,75 \\
\hline & PreQx acetaminofén & 14 & 87,50 \\
\hline & PreQx dipirona & 4 & 25,00 \\
\hline & PreQx corticoides & 5 & 31,25 \\
\hline
\end{tabular}

Variables cualitativas: $n$, frecuencia absoluta; \%, frecuencia relativa. Variables cuantitativas: m, mediana; RIC, rango intercuartílico; PreQx: prequirúrgico; AINEs: antiinflamatorios no esteroideos.

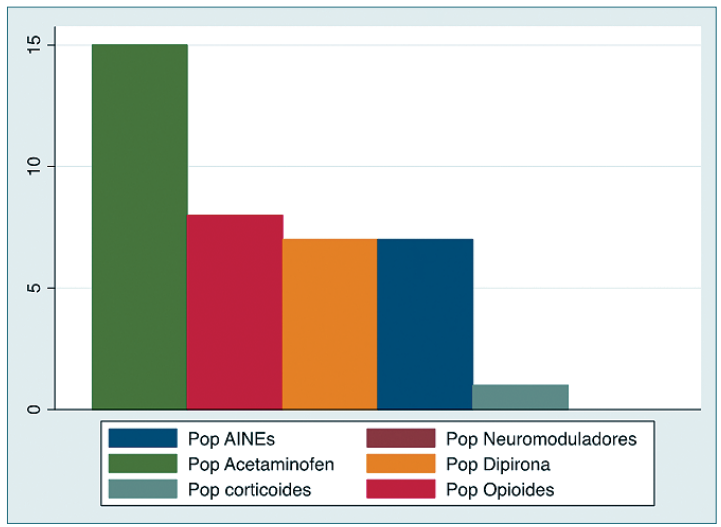

Figura 1. Frecuencia de la administración de medicamentos analgésicos posoperatorios. mina en dosis subanestésicas como alternativa a los opioides se demostró que su efecto no era superior, sin embargo, se determinó como un fármaco adecuado y de mayor seguridad en la analgesia multimodal, debido al menor riesgo de desarrollo de dependencia, en el manejo del dolor agudo POP[9]. Laskowski et al.[10], realizaron una revisión sistemática del uso de ketamina intraoperatoria en la que incluyeron estudios de 1966 a 2010 doble ciegos y controlados para un total de 4.701 pacientes en 47 estudios, encontrando como hallazgo significativo que la eficacia analgésica estaba dado en cirugía consideradas como dolorosas, mientras que en cirugías dentales, amigdalectomía y/o de cabeza y cuello no tuvo ningún beneficio adicional.

Estos hallazgos están relacionados con la interac- 


\begin{tabular}{|c|c|c|}
\hline \multicolumn{3}{|c|}{ Análisis descriptivo } \\
\hline Variable & $\mathrm{n} / \mathrm{m}$ & \%/RIC \\
\hline Náuseas & 3 & 18,75 \\
\hline Emesis & 2 & 12,50 \\
\hline Sedación & 0 & 0,00 \\
\hline Prurito & 0 & 0,00 \\
\hline Estreñimiento & 3 & 18,75 \\
\hline Desorientación & 0 & 0,00 \\
\hline Alucinaciones & 1 & 6,25 \\
\hline Hipertensión & 0 & 0,00 \\
\hline Taquicardia & 0 & 0,00 \\
\hline Otros & 0 & 0,00 \\
\hline Antiemético & 2 & 12,50 \\
\hline Laxante & 4 & 25,00 \\
\hline Fibra & 0 & 0,00 \\
\hline
\end{tabular}

Variables cualitativas: $\mathrm{n}$ frecuencia absoluta; \% frecuencia relativa. Variables cuantitativas: m mediana; RIC rango intercuartílico.

ción antagónica con el receptor de NMDA a nivel del cerebro y la médula espinal, que a su vez interviene en el aprendizaje, memoria y plasticidad sináptica, amplificación de las señales del dolor, desarrollo de la sensibilización central y la tolerancia a opioides[11]; además, interacciona con otros receptores: opioides, monoaminérgicos, colinérgicos, nicotínicos, muscarínicos y canales de sodio; y mejora de los sistemas antinociceptivos endógenos, bloqueando las señales aferentes de la vía espino-reticular y espino-talámica. Otro mecanismo productor de analgesia es la interacción con el transportador de serotonina aumentando su actividad central y la inhibitoria descendente relacionada con efectos antiinflamatorios mediados por la interleuquina 6[12].

Los resultados obtenidos en este estudio muestran una correlación con la disminución en la evaluación del dolor POP en las primeras 24 h, con calificaciones inferiores a 2 en tres cuartas partes de los pacientes, con disminución en el consumo de opioides con un promedio de 1 a 2 rescates. Esto acorde a un metaanálisis de 14 ensayos clínicos controlados con un total de 649 pacientes en POP de cirugía de columna, a quienes se les administró ketamina mostró una reducción del consumo de opioides hasta a $24 \mathrm{~h}$ con mínimos efectos adversos[13], con lo cual su utilización toma fuerza. Por su parte, Nielsen et al.[14] desarrollaron un ensayo clínico aleatorizado en pacientes llevados a cirugía de columna en el que, además de la disminución del dolor agudo, también mejoró el dolor crónico a 6 meses, encontraron que la disminución en el consumo de opioides acumulado en $24 \mathrm{~h}$ era de $42 \mathrm{mg}$ en el grupo que recibió ketamina comparados con el grupo que recibió placebo. Más adelante, un ensayo clínico realizado por Minosima et al.[15] en 2015 encontró que ketamina intra y posoperatorias hasta $48 \mathrm{~h}$ a dosis de $2 \mathrm{ug} / \mathrm{kg} / \mathrm{h}$ eran suficientes para disminuir el consumo de morfina, además de disminuir las náuseas y la emesis POP, con lo cual decreció la necesidad de antieméticos en estos pacientes favoreciendo el inicio temprano de la vía oral.

Con relación a la presentación de efectos adversos es de resaltar que los trabajos mencionados se han encontrado la disminución de la presentación de náuseas y vómito POP como principal beneficio en este aspecto[10]-[15], sin embargo, los efectos neuropsiquiátricos se vuelven más frecuentes cuando se usa el fármaco[10], esto último difiere de nuestro trabajo en tanto sólo 1 paciente presentó un episodio de alucinaciones visuales que resolvió de forma espontánea en un tiempo inferior a 5 minutos. La presentación de este efecto se encuentra relacionado con la dosis[11],[16]-[18]; sin embargo, en este caso la reacción puede ser explicada por una predisposición psíquica del paciente. Otros efectos adversos frecuentes son la taquicardia y la hipertensión, sin embargo, no se presentaron en ninguno de los pacientes.

Una limitación de este estudio fue el seguimiento 


\section{Tabla 4. Análisis descriptivo variables desenlaces específicas}

\begin{tabular}{|c|c|c|c|}
\hline \multicolumn{4}{|c|}{ Análisis descriptivo } \\
\hline Variable & $\mathrm{n} / \mathrm{m}$ & $\% /$ RIC & \\
\hline EVA Pop inmediato & 0 & $0-1$ & \\
\hline Dolor Pop inmediato & $\begin{array}{l}\text { Ausente } \\
\text { Leve } \\
\text { Moderado } \\
\text { Severo }\end{array}$ & $\begin{array}{r}12 \\
3 \\
0 \\
1\end{array}$ & $\begin{array}{r}75,00 \\
18,75 \\
6,25 \\
0,00\end{array}$ \\
\hline EVA Pop $1 \mathrm{~h}$ & 0 & $0-2$ & \\
\hline Dolor Pop $1 \mathrm{~h}$ & $\begin{array}{l}\text { Ausente } \\
\text { Leve } \\
\text { Moderado } \\
\text { Severo }\end{array}$ & $\begin{array}{l}9 \\
5 \\
0 \\
2\end{array}$ & $\begin{array}{r}56,25 \\
31,25 \\
0,00 \\
12,50\end{array}$ \\
\hline EVA Pop $4 \mathrm{~h}$ & 0 & $0-2$ & \\
\hline Dolor Pop $4 \mathrm{~h}$ & $\begin{array}{l}\text { Ausente } \\
\text { Leve } \\
\text { Moderado } \\
\text { Severo }\end{array}$ & $\begin{array}{l}9 \\
6 \\
1 \\
0\end{array}$ & $\begin{array}{r}56,25 \\
37,50 \\
6,25 \\
0,0\end{array}$ \\
\hline EVA Pop $12 \mathrm{~h}$ & 0 & $0-2$ & \\
\hline Dolor Pop 12 horas & $\begin{array}{l}\text { Ausente } \\
\text { Leve } \\
\text { Moderado } \\
\text { Severo }\end{array}$ & $\begin{array}{r}11 \\
4 \\
1 \\
0\end{array}$ & $\begin{array}{r}68,75 \\
25,00 \\
6,25 \\
0,00\end{array}$ \\
\hline EVA Pop $24 \mathrm{~h}$ & 1 & $0-2$ & \\
\hline Dolor Pop $24 \mathrm{~h}$ & $\begin{array}{l}\text { Ausente } \\
\text { Leve } \\
\text { Moderado } \\
\text { Severo }\end{array}$ & $\begin{array}{l}8 \\
7 \\
1 \\
0\end{array}$ & $\begin{array}{r}50,00 \\
43,75 \\
6,25 \\
0,00\end{array}$ \\
\hline Control adecuado dolor $24 \mathrm{~h}$ & 14 & 87,50 & \\
\hline Satisfacción (0-10) & 10 & $8,5-10$ & \\
\hline Inicio movilización & $\begin{array}{l}1 \text { día } \\
2 \text { días }\end{array}$ & $\begin{array}{r}14 \\
2\end{array}$ & $\begin{array}{l}87,50 \\
12,50\end{array}$ \\
\hline
\end{tabular}

Variables cualitativas: $\mathrm{n}$ frecuencia absoluta; \% frecuencia relativa. Variables cuantitativas: $\mathrm{m}$ mediana; RIC rango intercuartílico. EVA: escala verbal análoga. Pop: posoperatorio.

realizado sólo durante $24 \mathrm{~h}$ evaluando el dolor agudo en ese tiempo, por lo que no puede determinarse si a largo plazo los pacientes tenían mejoría del dolor o desarrollo de dolor crónico, para lo cual haría falta mayor investigación en el campo.

\section{Conclusión}

El seguimiento a los pacientes sometidos a artrodesis de columna que recibieron un protocolo de analgesia con infusión de ketamina muestra un control adecuado y eficaz del dolor agudo POP, con mínimos eventos adversos y una satisfacción adecuada por parte de los pacientes. Ante estos hallazgos y la evidencia de su uso para este tipo de procedimiento, se sugiere la continuación del protocolo instaurado en la institución y no descartar la posibilidad de ampliar su uso en procedimiento que se prevea altas calificaciones de dolor en el POP.

Agradecimientos: Los autores agradecen en primer lugar a los pacientes participantes del estudio por su colaboración en la producción de conocimiento científico. En segundo lugar, al Hospital Hernando Moncaleano Perdomo (Neiva, Colombia) y al personal médico y de enfermería, por su disposición. 


\section{Referencias}

1. Practice Guidelines for Acute Pain Management in the Perioperative Setting. An updated Report by the American Society of Anesthesiologist Task Force on Acute Pain Management. Anesthesiology. [Internet]. 2012 Vol. 116, 248-273. [citado 2019 Jul 06]; 43(1):16-22. Disponible en: https://revistachilenadeanestesia.cl/rocuronio-en-obesosdebe-dosificarse-por-peso-real-opeso-ideal/]

2. eddi D, Curran N. Chronic pain after surgery: pathophysiology, risk factors and prevention. Postgrad Med J. 2014 Apr;90(1062):222-7. https://doi.org/10.1136/ postgradmedj-2013-132215 PMID:24572639

3. Martínez IM, Portero V. Estándares de uso adecuado de la artrodesis vertebral. Sevilla: Agencia de Evaluación de Tecnologías Sanitarias de Andalucía; 2009.

4. e la Torre M, Martínez-Quiñones JV. Artrodesis lumbar posterior. A propósito de 89 casos. Neurocirugia (Astur). 1997;8(3):21120. https://doi.org/10.1016/ S1130-1473(97)71032-7.

5. Bassols A, Bosch F, Baños JE. ¿Cómo trata la población en general su dolor? Una encuesta en Cataluña, España. J Manejo de síntomas de dolor. 2002; 23 (4): 318-328.

6. Dueñas M, Ojeda B, Salazar A, Mico JA, Failde I. A review of chronic pain impact in patients their social enviroment and the heatl care system. J Pain Res. 2016; 9: 457-467. Publicado el 28 de junio de 2016. https://doi. org/10.2147/JPR.S105892.

7. Gan TJ. Poorly controlled postoperative pain: prevalence, consequences, and prevention. J
Pain Res. 2017;10: 2287-2298. Published 2017 Sep 25. https:// doi.org/10.2147/JPR.S144066.

8. Manchado-Alba JE, RamirezSarmiento JO, Salazar-Ocampo DF. Estudio multicéntrico sobre efectividad de control del dolor posquirúrgico en pacientes de Colombia. Colombian Journal of Anesthesiology. 2016 AprJun;44(2):114-20. https://doi. org/10.1016/j.rca.2016.02.004.

9. Karlow N, Schlaepfer $\mathrm{CH}$, Stoll $C R$, Doering $M$, Carpenter $C R$, Colditz GA, et al. A systematic review and meta-analysis of ketamine as alternative to opioids for acute pain in the emergency department. Acad Emerg Med. 2018 Oct;25(10):1086-97. https:// doi.org/10.1111/acem.13502 PMID:30019434

10. Laskowski K, Stirling A, McKay WP, Lim HJ. A systematic review of intravenous ketamine for postoperative analgesia. Can J Anaesth. 2011 Oct;58(10):911-23. https://doi. org/10.1007/s12630-011-9560O PMID:21773855

11. Bell RF, Kalso EA. Ketamine for pain management. Pain Rep. 2018;3(5):e674. Published 2018 Aug 9. https://doi.org/10.1097/ PR9.0000000000000674.

12. Mion G, Villevieille T. Ketamine pharmacology: an update (pharmacodynamics and molecular aspects, recent findings). CNS Neurosci Ther. 2013 Jun;19(6):370-80. https:// doi.org/10.1111/cns.12099 PMID:23575437

13. Pendi A, Field R, Farhan SD, Eichler M, Bederman SS. Perioperative Ketamine for Analgesia in Spine Surgery: A Meta-analysis of Randomized Controlled Trials. Spine. 2018 Mar;43(5):E299-307. https://doi.org/10.1097/

BRS.0000000000002318 PMID:28700455

14. Nielsen RV, Fomsgaard JS, Siegel $H$, Martusevicius R, Nikolajsen L, Dahl JB, et al. Intraoperative ketamine reduces immediate postoperative opioid consumption after spinal fusion surgery in chronic pain patients with opioid dependency: a randomized, blinded trial. Pain. 2017 Mar;158(3):463-70. https://doi.org/10.1097/j. pain.0000000000000782 PMID:28067693

15. Minoshima R, Kosugi S, Nishimura D, Ihara N, Seki H, Yamada $\mathrm{T}$, et al. Intra- and postoperative low-dose ketamine for adolescent idiopathic scoliosis surgery: a randomized controlled trial. Acta Anaesthesiol Scand. 2015 Nov;59(10):1260-8. https:// doi.org/10.1111/aas. 12571 PMID:26079533

16. Quibell $R$, Prommer EE, Mihalyo $M$, Twycross R, Wilcock A. Ketamine*. J Pain Symptom Manage. 2011 Mar;41(3):640-9. https://doi.org/10.1016/j. jpainsymman.2011.01.001 PMID:21419322

17. Rivera $D$, Susunaga $P$, Pérez M. Ketamina: estado del Arte. Revista Facultad de Salud RFS. 2013;5(1):83. https://doi. org/10.25054/rfs.v5i1.134.

18. Neira Reina F, Ortega García JL. La ketamina en el tratamiento del dolor crónico según medicina basada en la evidencia. Rev. Soc. Esp. Dolor [Internet]. 2016 Dic [citado 2020 Ago 14]; 23(6): 292-306. Disponible en: http://scielo. isciii.es/scielo. php?script=sci_ arttext\&pid=\$1134$80462016000600006 \&$ lng=es https://doi.org/10.20986/resed.2016.3462/2016. 